\title{
Philosophiques
}

\section{Inconscient social de la psychanalyse et points aveugles du psychanalyste}

\section{Ghyslain Charron}

Volume 4, numéro 2, octobre 1977

Philosophie et psychologie

URI : https://id.erudit.org/iderudit/203080ar

DOI : https://doi.org/10.7202/203080ar

Aller au sommaire du numéro

Éditeur(s)

Société de philosophie du Québec

ISSN

0316-2923 (imprimé)

1492-1391 (numérique)

Découvrir la revue

Citer cet article

Charron, G. (1977). Inconscient social de la psychanalyse et points aveugles du psychanalyste. Philosophiques, 4(2), 293-304. https://doi.org/10.7202/203080ar d'utilisation que vous pouvez consulter en ligne.

https://apropos.erudit.org/fr/usagers/politique-dutilisation/ 


\title{
INCONSCIENT SOCIAL DE LA PSYCHANALYSE ET POINTS AVEUGLES DU PSYCHANALYSTE
}

\author{
par Ghyslain Charron
}

Je centrerai ma réflexion sur deux questions soulevées par $\mathrm{R}$. Castel dans son ouvrage Le psychanalysme $e^{1}$ : l'inconscient social impliqué dans la neutralité analytique; les effets de méconnaissance produits par cette attitude, entre autres, la prétention à l'autonomie à l'égard du socio-politique. Après avoir exposé la pensée de Castel à ce sujet, je voudrais défendre trois thèses : la psychanalyse n'a pas les outils conceptuels pour penser les institutions et son rapport au pouvoir ; comme il n'y a pas d'explication psychogénétique des institutions sociales et politiques, le psychanalyste est obligé de recourir à une autre discipline s'il veut comprendre la psychanalyse comme institution; il n'y a pas de synthèse possible du social et du psychologique.

La psychanalyse ouvre une nouvelle scène, celle de l'inconscient, mais cette opération est en même temps travail d'occultation. R. Castel appelle psychanalysme, l'effet-psychanalyse immédiat produit par le parti-pris de la théorie et de la pratique analytiques à l'égard de la réalité socio-politique. La relation entre la psychanalyse et le psychanalysme est différente de celle qui unit un savoir à son idéologisation, car le psychanalysme est le processus d'idéologisation produit par la psychanalyse elle-même et non par " des altérations subies par elle en dehors de son domaine légitime" (p. 11). Le psychanalysme est l'implication socio-politique directe de la méconnaissance du socio-politique. Par l'abstraction inhérente à son point de vue, la psychanalyse produit des effets qui lui demeurent opaques dans leur spécificité, même si elle discourt à leur sujet. Son aveuglement concerne en tout premier lieu ce qui est au principe de son pouvoir et son rapport au pouvoir.

1. R. CASTEL, Le psychanalysme, Éd. Maspero, textes à l'appui/psychiatrie, 1973, 288 p. 
Castel avance trois propositions fondamentales:

a) Le rapport de la psychanalyse à ses usages même les plus "dévoyés" n'est jamais une relation de pure extériorité.

b) La relation analytique la plus épurée a immédiatement des effets sociaux spécifiques qui ne sont jamais socialement neutres.

c) Le rapport entre a) et b) permet de comprendre de l'intérieur de son dispositif la place occupée aujourd'hui par la psychanalyse parmi les idéologies dominantes et les institutions de contrôle social (p. 18).

C'est en articulant ces trois points que Castel compte faire voir la logique interne du psychanalysme. Sa problématique tourne autour des questions suivantes : quel est dans le procès de sa production le rapport du savoir analytique au pouvoir socio-politique? quel est dans le champ de son exploitation sociale le rapport du savoir analytique au pouvoir socio-politique? (p. 235). Il adopte le point de vue du socio-historien et se propose de traiter la psychanalyse comme une institution parmi d'autres dans l'ensemble des institutions constituant l'ordre socio-politique. Partant du rituel analytique en tant qu'il instaure la psychanalyse comme le dispositif de l'accès à l'inconscient, Castel montre que le mécanisme de la neutralisation inhérent au contrat qui fonde le rituel entraîne nécessairement un effet politique, à savoir l'apolitisme. Il se met ensuite à la recherche des conditions qui instituent le rituel luimême, conditions méconnues par la psychanalyse et que, pour ce motif, il appelle inconscient de la psychanalyse, plus précisément son inconscient social. L'intention de Castel est de montrer les conséquences du rituel et du contrat qui le fondent. Si l'on sait reconnaître dans la psychanalyse un contrat de tutelle, alors on peut apercevoir l'inscription de la psychanalyse dans les structures de pouvoir et élaborer une analyse socio-politique de la psychanalyse comme opérateur social spécifique. Nous ne retiendrons ici de l'ouvrage de Castel que certaines idées concernant la structure du rituel analytique, l'inconscient social de ce rituel et les relations entre psychanalyse et psychanalysme qui en découlent.

C'est en effet par un contrat ou une convention que s'instaure la situation où deux individus (l'analyste et l'analysant) poursuivront cette tâche qu'on nomme une psychanalyse. Le processus 
analytique lui-même ne saurait s'effectuer sans cette convention de départ. Il importe immédiatement de remarquer que le dispositif à la base du corpus analytique (théorie et pratique) met " hors jeu les déterminismes politiques et sociaux au quadruple niveau de son instauration (le contrat analytique), de son déroulement (la relation duelle), de ses matériaux (les formations de l'inconscient), de ses concepts (les catégories du discours analytique)" (p. 39). La psychanalyse n'a pu commencer à exister et se fonder comme savoir et pratique spécifiques qu'en forgeant son propre système de référence. C'est à l'intérieur de cet espace que le processus d'exploration de l'inconscient pourra s'effectuer. Le dispositif technique de la psychanalyse "opère en imposant une suspension de la réalité, une neutralisation de ce qui, dans l'existence ordinaire, n'est jamais neutre. En empruntant provisoirement à Serge Leclaire une distinction commode, on dira que la convention analytique met hors jeu la réalité (socio-politique) pour démasquer le réel (analytique) ».(p. 41). Toute la question est bien de savoir ce qu'il advient de cette réalité que l'analyste met entre parenthèses afin d'accomplir son travail. Elle n'est pas éliminée pour autant, estime Castel. Cette réalité n'est pas devenue extérieure à la situation analytique, elle demeure présente dans la relation analytique mais "neutralisée, c'est-à-dire travestie et devenue méconnaissable ". Bien sûr, les conditions du contrat analytique (adhésion à la règle fondamentale, respect du protocole) instaurent une coupure avec la réalité socio-politique telle que vécue dans la vie ordinaire, et ces conditions constituent un champ nouveau en le clôturant. C'est à l'intérieur de ce champ expérimental que par la médiation de la parole apparaîtront les fantasmes et à travers eux les voeux inconscients et leurs conflits. La technique de l'association libre, écoute flottante et interprétation, n'a, semble-t-il, rien à voir avec l'action politique. Le passage à l'acte est du point de vue analytique non un accomplissement mais un échec, car ce qui est recherché, c'est la reconnaissance des conflits psychiques inconscients et leur dénouement. Le passage à l'acte ne fait que perpétuer la méconnaissance des conflits inconscients. De même, si au lieu d'écouter, d'analyser son contre-transfert et d'interpréter ce que l'analysant lui raconte, l'analyste se lance dans un discours analogue à ceux que tiennent nos hommes politiques en temps d'élection, il va manifestement à l'encontre du contrat qui le lie à son patient. Tout cela est incontestable, répondra Castel, mais la question à poser est celle-ci ; 
comment est constitué ce personnage qu'on appelle le psychanalyste "neutre " et quelles sont les implications de cette neutralité ? Cette convention de neutralité est justement, d'après Castel, lourde d'ambiguité. La neutralisation est d'abord une exigence technique. En effet, l'analyste doit, pour que le travail analytique s'accomplisse, se faire support " neutre " de fantasmes, surface de projection, objet de transfert. Il n'en reste pas moins, souligne Castel, qu'une position de pouvoir est ici impliquée même si l'analyste ne transgresse jamais la convention de neutralité. "Techniquement parlant, la neutralité analytique est une condition de possibilité du transfert ; politiquement parlant, c'est l'incarnation de la politique de l'apolitisme" (p. 50). La psychanalyse ne peut avec les concepts et les méthodes dont elle dispose analyser la signification extra-analytique de sa neutralité, distinguer entre idéologies dominantes et idéologies dominées, etc. Il reste que l'analyste, par sa pratique, s'inscrit quelque part dans un rapport de forces et occupe une position dans la société. Quelles qu'en soient les raisons, " en tant qu'il a le pouvoir, le processus analytique neutralise. Il reproduit le pouvoir neutralisant du psychanalyste " neutre " " (p. 57). Tel est un élément essentiel du rituel analytique.

Comme le garagiste, le plombier ou l'avocat, le psychanalyste entre dans "une relation de service personnalisée " lorsqu'il accepte de prendre quelqu'un en traitement. Ce dernier demande au technicien de réparer ce qui va mal dans sa vie. Les règles du contrat analytique construisent une sorte de laboratoire monté exprès pour produire un effet spécifique, disons, pour faire vite, le dénouement des conflits inconscients qui paralysent l'individu et l'empêchent de vivre. Rappelons-nous, dit Castel, la remarque de Durkheim : "Tout n'est pas contractuel dans le contrat. " Le contrat analytique comme tous les autres " exige des préréquisits sociaux, politiques, économiques, culturels, faute desquels la relation analytique ne pourrait être tenue, ni tenir sa gageure. Mais ils sont inscrits dans sa structure sans être donnés dans sa formule " (p. 59). Et c'est précisément parce qu'ils ne sont pas donnés dans sa formule qu'ils ne peuvent être reconnus dans leur poids extraanalytique comme élément de la réalité socio-politique. Bien sûr, cet extra-analytique, condition effective de possibilité de l'entreprise analytique, se retrouve dans la relation analytique elle-même, mais il est réinterprété, chargé de significations nouvelles et deve- 
nues opératoires dans et pour le dispositif analytique. Ainsi l'argent prendra un sens symbolique, la neutralité politique sera justifiée par la théorie du transfert. Ce qui est barré par la convention analytique représente essentiellement la neutralisation des données qui constituent la problématique socio-politique du pouvoir (p. 60). Le paradoxe est que, tout en supposant et réitérant dans son travail les oppositions de la structure sociale (privé/public, parole/acte, corps comme support des fantasmes/corps comme instrument de travail), l'analyste prétende accéder à l'inconscient de l'individu seulement s'il neutralise ces oppositions. Cela même que le dispositif analytique exclut pour exister, Castel l'appelle "inconscient social de la psychanalyse $»$.

Le terme inconscient est ici employé à la suite de Freud au sens de refoulé, oublié, non reconnu ou mieux méconnu, c'est-àdire à la fois présent et travesti dans les symptômes. En tant que socio-historien, Castel considère comme un symptôme le discours de l'analyste et estime qu'en partie au moins ce discours fonctionne comme rationalisation. Dans cette mesure, son contenu manifeste exprime - dissimule les raisons réelles qui le soutiennent et l'alimentent. Pourtant, l'analyse qu'entend mener Castel ne veut rien emprunter aux catégories et aux méthodes de la psychanalyse, car l'inconscient qu'il cherche à mettre au jour ne "parle pas le langage de la pulsion ni du désir ", mais plutôt celui du pouvoir socio-politique. C'est pourquoi cet inconscient est dit social. La tâche d'une sociologie critique consistera à dépister les éléments de la réalité sociale qui travaillent souterrainement le réel analytique. Ainsi la neutralité et le pouvoir de l'analyste sont autant d'éléments de cette sorte.

La première question du socio-historien analysant l'inconscient social de la psychanalyse concerne les effets produits par la méconnaissance des conditions socio-historiques inhérentes au dispositif analytique. Castel relève au moins trois couches de méconnaissance :

a) au niveau phénoménologique : dans l'environnement de la relation analytique;

b) au niveau structural : dans l'économie de cette relation;

c) au niveau du discours analytique: dans l'explicitation théorique de la relation en question. 
Le premier niveau est le plus extérieur au dispositif lui-même. Il concerne tout ce qui se rattache au statut social du psychanalyste. Par sa fonction même, l'analyste occupe une position dans l'échiquier social et économique. Une étude de type ethnologique du milieu analytique et du rapport analyste-analysant serait nécessaire ici. Il s'agirait de dégager les invariants qui organisent ces positions et ces relations. Au second niveau, on analyserait l'échange qui relie un sujet supposé savoir et un client porteur d'une demande dans un projet de transformation. On verrait que les notions de spécialiste compétent, de contrat, d'honoraires prennent en psychanalyse un sens spécifique. Ainsi le psychanalyste "inclut la nécessité du paiement dans le contrat, faute duquel la totalité de la relation est invalidée "( p. 72). Le paiement par l'analysant luimême est comme une condition nécessaire à l'efficacité d'une cure. Par ailleurs, l'intervention effectuée par un chirurgien produira les mêmes résultats, que le médecin soit payé par le patient, payé par une institution ou pas payé du tout. Une sociologie critique de la psychanalyse devrait aussi montrer comment la méconnaissance des implications sociales et politiques joue même au niveau du discours analytique lorsque l'analyste entreprend de penser théoriquement la relation analytique. Ce que Castel reproche à la psychanalyse, ce n'est pas tant sa complicité avec les structures sociopolitiques du pouvoir (reproche-t-on à une pierre de tomber?), c'est plutôt de prétendre s'en affranchir et de jouer à l'autonomie ou à la subversion (p. 78). L'inconscient social de la psychanalyse, c'est précisément ce qu'elle méconnaît quant à sa relation aux structures socio-politiques du pouvoir. Cette méconnaissance n'est pas un accident de parcours. Elle tient au fait que les catégories du discours analytique ne peuvent rendre compte des conditions sociales qui rendent possible le discours analytique lui-même. Si la tâche de la psychanalyse est d'explorer l'inconscient psychique individuel, on pourrait dire par analogie que la fonction de la sociologie critique de la psychanalyse sera d'analyser les mécanismes et les effets de l'inconscient social de la psychanalyse comme institution, comme pratique et comme discours théorique. C'est l'existence chez le psychanalyste de points aveugles qui fait que la psychanalyse a partie liée avec le psychanalysme.

Si la psychanalyse "est la pratique de la totalité de ses effets ", c'est elle-même qui produit ce processus d'idéologisation que Castel appelle le psychanalysme. La psychanalyse n'est pas une 
idéologie parmi d'autres. Elle est plutôt une discipline qui produit en même temps des effets de connaissance et des effets idéologiques, c'est-à-dire de méconnaissance. La thèse de Castel sur la logique du psychanalysme se formule en deux propositions essentielles :

a) L'extra-analytique est présent dans le dispositif analytique, mais sous une forme en général méconnue ;

b) Cette méconnaissance n'est pas une simple ignorance, ni une pure occultation mais une réinterprétation unilatérale (partielle et partiale), directement porteuse d'effets sociaux également unilatéraux, par exemple l'apolitisme (p. 205).

Comme nous l'avons vu, Castel distingue trois niveaux de méconnaissance, c'est-à-dire trois couches d'idéologisation. Ces productions idéologiques sont à la fois " spécifiques à la psychanalyse, nécessaires et liées entre elles. C'est pourquoi on peut parler à leur propos de logique, et de la logique d'un processus produit par (et non subi par) la psychanalyse: le psychanalysme" (p. 205).

L'ouvrage L'Univers contestataire est une belle illustration d'idéologisation du premier niveau. Ici le modèle d'interprétation fonctionne sur un régime totalitaire. On suppose que tout est psychanalysable. Mais cette prétention n'est pas le fruit du hasard. Elle est rendue possible par le fait qu'il n'y a pas de théorie analytique rigoureuse des conditions d'exportation du schéma analysteanalysant. Pour saisir la différence entre la théorie des formations de l'inconscient et la théorie des conditions matérielles et sociales du contrat analytique, il faut changer de système de référence, recourir à un système de référence qu'on ne peut déduire du système de référence analytique. Ceci pour la bonne raison que la psychanalyse ne possède pas en elle-même de catégories pour penser le pouvoir, le social et le politique dans leurs dimensions non psychiques. Mais, comme le dit Castel, "si le butoir n'est pas dans la ligne du dispositif analytique, un modèle d'interprétation analytique est virtuellement d'emblée un modèle d'interprétation totalitaire" (p. 206). En effet, s'il n'y a pas à l'intérieur de la théorie un principe de démarcation, rien n'empêche d'interpréter en termes de conflits intrapsychiques, de résistance, de projection, tout rapport socio-politique et toute question de pouvoir. La pratique analytique elle-même entraîne une autre couche d'idéologisation, car dans la 
mesure même où elle impose les règles de sa convention, elle invalide nécessairement la problématique des investissements inconscients. Et le projet de comprendre tous les enjeux de l'existence, à partir de leur signification pour l'économie inconsciente du sujet, conduit la psychanalyse à cette idéologisation qui consiste à réduire les déterminismes socio-politiques à des facteurs psychiques individuels et interindividuels (pp. 209-210).

Telle est en gros, l'idée que Castel se fait du rituel analytique, des conditions de son application et des résultats qu'il produit. Je n'ai retenu ici qu'une des tâches que s'assigne Castel, celle de " déplacer la question des responsabilités sociales de la psychanalyse de la périphérie de ses usages au centre de son dispositif ", pour montrer que par la convention même qui la constitue, la psychanalyse se rend complice du système socio-économique dans lequel elle s'inscrit. Une autre tâche, dont nous n'avons pas parlé, consisterait à faire une étude historique de la pénétration de la psychanalyse dans diverses institutions et de voir comment elle imprime ses finalités dans la vie sociale et comment elle contribue au contrôle social. Nous nous contenterons pour l'instant de réfléchir brièvement sur le pivot autour duquel s'articule la critique de Castel à l'égard de la psychanalyse comme institution et comme pratique sociale.

La condition d'efficacité d'une pratique et la condition de sens d'un discours est un ensemble de postulats et de règles qui donnent prise sur une réalité selon une perspective déterminée. La psychanalyse n'échappe pas à ce principe qui vaut pour toutes les disciplines. Adopter le point de vue psychanalytique, c'est exclure le point de vue du socio-politicien et vice-versa. L'épistémologue, lui, s'interroge sur le statut des théories et des discours, sur les implications de ce statut, sur les rapports entre les diverses disciplines. Cette multiplicité de disciplines pourrait être vue comme un mal provisoire pour celui qui croit à une sorte de savoir dernier, synthèse absolue et sans point de vue. Si un tel savoir existait, il devrait articuler les deux propositions suivantes ou choisir entre elles si elles sont contradictoires : a) la neutralité analytique est condition de possibilité du transfert ; b) la neutralité analytique est l'incarnation de la politique de l'apolitisme. D'emblée, je dirai que me parait impossible une science qui serait synthèse du point de vue psychanalytique et du point de vue socio-politique. On com- 
prendra mieux pourquoi quand j'aurai explicité les trois thèses que j'entends soutenir.

Première thèse : la psychanalyse n'a pas les outils conceptuels pour penser les institutions et son rapport au pouvoir. Les concepts freudiens ont été construits pour décrire et expliquer les avatars du désir et des fantasmes, le jeu des représentations, des affects et des intentions, bref la réalité psychique telle qu'elle se dévoile dans la situation transférentielle et contre-transférentielle de l'analyse. Il ne faut donc pas s'étonner que nous ne trouvions pas dans l'oeuvre freudienne une problématique socio-politique comme telle. La question se pose cependant de savoir " par quoi la psychanalyse est parlée, sans pouvoir le parler : à savoir la dimension politique de sa pratique. Ce non-dit de la psychanalyse revient à insister comme symptôme au lieu même où s'établit son articulation avec le système politique, au niveau où le langage politique s'actualise : c'està-dire au niveau de la psychanalyse comme institution, ayant une fonction précise dans un ensemble politique précis ${ }^{2}$. Ici, seules les investigations empiriques peuvent fournir des réponses. Il revient au sociologue d'imaginer des hypothèses et de les tester. $\grave{A}$ moins de postuler des principes de fonctionnement identiques dans les institutions et dans la psyché, il faut bien admettre que le psychanalyste comme tel n'a pas les catégories pour penser son rapport à l'institution qu'est la psychanalyse et comprendre les relations entre cette institution et les autres institutions socio-politiques.

Cela me conduit à ma deuxième thèse : il n'y a pas d'explication psychogénétique des institutions sociales et politiques. Psyché et société sont inséparables et irréductibles l'une à l'autre, on ne peut expliquer l'une par l'autre. Comme le dit C. Castoriadis : "Ce que l'individu peut produire, c'est des phantasmes privés, non pas des institutions ${ }^{3}$. Le psychanalyste est habilité à

2. F. Gantheret, "Freud et la question socio-politique ", dans Partisans, février-mars 1969, no 46, p. 16

3. C. Castoriadis, L'institution imaginaire de la société, Seuil, 1975, p. 202. Comme le dit Castoriadis, les solutions que Freud apportait au problème de la socialisation de la psyché " restaient mythologiques du fait qu'il croyait pouvoir déduire l'institution à partir du fonctionnement psychique" (p. 417). "La perspective psychogénétique à elle seule est radicalement incapable de rendre compte de la formation de l'individu social, du processus de socialisation de la psyché. Truisme, que l'écrasante majorité des psychanalystes - à commencer par Freud lui-même - s'obstine à ignorer. Ce qui commande cette obstination, et l'occultation du social-historique qui va de pair avec elle, est la tenace illusion de la possibilité de réduire le psychique au biologique [. . . ] elle-même commandée par la volonté d'éliminer l'imaginaire, aussi bien comme imaginaire social que comme imagination radicale de la psyché " (ibid., p. 426) 
interpréter ces phantasmes. Mais dans la mesure où les institutions ne sont pas réductibles à des phantasmes, l'analyste est obligé de recourir à d'autres disciplines s'il veut comprendre la psychanalyse comme institution.

Ma troisième et dernière thèse est que, s'il y a des recherches pluridisciplinaires, il ne peut y avoir de discipline qui soit synthèse du social et du psychologique. Je rejoins ici la théorie de G. Devereux. Je dirai avec lui que la recherche pluridisciplinaire n'a rien à voir avec une interdisciplinarité "de type additif, fusionnant, synthétique ou parallèle $"^{4}$. Le rapport entre le cadre de référence et le discours du sociologue et le cadre de référence du psychanalyste en est un de complémentarité au sens entendu par Devereux ; ce qui implique que les deux discours ne peuvent être tenus simultanément et ce qui entraîne l'impossibilité logique d'une synthèse entre les deux.

Si nous supposons vraies ces trois thèses, quelles conséquences en tirer en ce qui concerne la soi-disant neutralité analytique? Le psychanalyste décrit celle-ci comme la condition du transfert, le sociologue Castel la voit comme l'incarnation de la politique de l'apolitisme, comme impliquant un inconscient social et entraînant cette méconnaissance spécifique qu'est la prétention d'autonomie à l'égard du socio-politique. Comment savoir qui a raison ? Peuventils avoir raison tous les deux?

$\grave{A}$ mon avis, le psychanalyste ne peut trouver dans sa discipline les critères et les catégories pour juger du bien-fondé ou de la fausseté de la proposition de Castel au sujet de l'apolitisme. Seules les recherches empiriques (sociologiques et historiques) peuvent corroborer ou infirmer l'hypothèse que les analystes et les analysés deviennent apolitiques en raison de leur expérience de la psycha. nalyse. En attendant, l'analyste peut toujours répondre que dans la situation analytique, c'est à l'ordre du désir et du fantasme qu'il a affaire. Le psychanalyste ne peut en même temps explorer la psyché de son patient pour en mettre à jour les conflits et convertir ce même individu à telle idéologie politique, ou l'inciter à s'engager dans tel parti. Si essayer de convaincre, c'est empêcher la formation et l'analyse du transfert, instrument indispensable de l'exercice du métier d'analyste, il faut choisir entre agir politi-

4. G. Devereux, Ethnopsychanalyse complémentariste, Flammarion, 1972, p. 10. 
quement et pratiquer l'analyse'. Quant à la réalité socio-politique, le psychanalyste au travail est par rapport à elle comme par rapport à la réalité biologique, il sait qu'elle existe, mais il ne peut avec les outils qu'il emploie ni la connaître ni la transformer. Il est indéniable que l'analyse est pour l'analysant investie d'un pouvoir. Sans quoi, ses interventions n'auraient aucun poids et aucune efficacité. Mais dans la mesure justement où l'analyste réussit, le "sujet supposé savoir "qu'était l'analyste durant le traitement est aboli à la fin. Loin de conduire à l'apolitisme, la neutralité de l'analyste et la liquidation du transfert devraient théoriquement rendre le sujet plus disponible pour une conscience sociale et politique plus aiguë. Il revient aux investigations empiriques de dire si tel est effectivement le cas et dans quelle mesure.

Quant à l'expression de Castel " inconscient social de la psychanalyse ", elle ne me paraît pas très heureuse. Comment la psychanalyse comme discipline peut-elle avoir au sens strict un inconscient? Peut-on vraiment attribuer à une discipline le mécanisme du refoulement? Si une connaissance totale est une contradiction dans les termes, on serait mal venu de reprocher à une discipline de ne pas parler de ce qui ne peut être aperçu et dit que par un autre savoir en fonction d'un autre cadre de référence. Au lieu d'attribuer à la psychanalyse un inconscient, je trouverais plus juste de dire que les psychanalystes ont des points aveugles. Ils en ont d'autant plus qu'ils méconnaissent davantage les présupposés de leur cadre de référence et, partant, les limites de leurs explications. Et comment reconnaître ces présupposés et leurs implications? Par le recours à d'autres disciplines : l'histoire, la sociologie et, pourquoi pas? la réflexion épistémologique. Puisque la réflexion totale est impossible, comme chacun sait, les points aveugles dureront autant que l'homme, mais il y a cependant moyen de limiter les dégâts. Dans la mesure même où l'auto-analyse et l'esprit critique contrebalanceront la naïveté et la bonne conscience. Il est certain que si l'analyste estime inévitable et comme an-historique la réalité sociale et politique où baigne sa pratique, il ne peut faire autrement que d'induire plus ou moins subrepticement chez ses analysés le conformisme socio-politique.

De l'avis de bien des analystes, la psychanalyse serait une pra-

5. Pour une réflexion plus approfondie sur ce qu'on nomme communément " la neutralité de l'analyste ", voir S. LeCLAIRE, Psychanalyser, Ed. du Seuil, 1968, pp. 9.27. 
tique révolutionnaire et même subversive. Je ne sais dans quelle mesure ils ont raison, je sais seulement par l'ouvrage d'A. Stéphane, L'univers contestataire, que la psychanalyse n'est pas un remède absolument efficace pour l'abolition des préjugés chez le psychanalyste. T. Reik, qui en avait rencontré plus d'un, ne disaitil pas : "Il est beaucoup plus facile de faire rentrer une idée nouvelle dans la tête de la Statue de la Liberté que dans l'esprit de bon nombre de psychanalystes. "Pourtant je me refuse à suivre Castel quand il soutient que « le psychanalyste produit l'apolitique comme le boulanger fabrique le pain ", tant que des enquêtes détaillées et approfondies n'auront pas montré qu'il en est bien ainsi.

Faculté de Philosophie, Université d'Ottawa. 